\title{
The adsorption of benzene on copper, silver, and gold surfaces
}

\author{
Ante Bilić, Jeffrey R. Reimers \\ School of Chemistry, The University of Sydney, NSW 2006, Australia
}

Noel S. Hush

School of Chemistry, The University of Sydney, NSW 2006, Australia

School of Molecular and Microbial Biosciences, The University of Sydney, NSW 2006, Australia

Rainer C. Hoft, Michael J. Ford

Institute for Nanoscale Technology, University of Technology Sydney,P.O. Box 123, Broadway, NSW 2007, Australia

\begin{abstract}
The adsorption of benzene on the $\mathrm{Cu}(111), \mathrm{Ag}(111), \mathrm{Au}(111)$ and $\mathrm{Cu}(110)$ surfaces at low coverage is modelled using density-functional theory (DFT) using periodicslab models of the surfaces, as well as using both DFT and complete-active-space self-consistent field theory with second order Møller-Plesset perturbation corrections (CASPT2) for the interaction of benzene with a $\mathrm{Cu}_{13}$ cluster model for the $\mathrm{Cu}(110)$ surface. For the binding to the (111) surfaces, key qualitative features of the results such as weak physisorption, the relative orientation of the adsorbate on the surface, and surface potential changes are in good agreement with experimental findings. Also, the binding to $\mathrm{Cu}(110)$ is predicted to be much stronger than that to $\mathrm{Cu}(111)$ and much weaker than that seen in previous calculations for $\mathrm{Ni}(110)$, as observed. However, a range of physisorptive-like and chemisorptive-like structures are found
\end{abstract}


for benzene on $\mathrm{Cu}(110)$ that are roughly consistent with observed spectroscopic data, with these structures differing dramatically in geometry but trivially in energy. For all systems, the bonding is found to be purely dispersive in nature with minimal covalent character. As dispersive energies are reproduced very poorly by DFT, the calculated binding energies are found to dramatically underestimate the observed ones, while CASPT2 calculations indicate that there is no binding at the HartreeFock level and demonstrate that the expected intermolecular correlation (dispersive) energy is of the correct order to explain the experimental binding-energy data. DFT calculations performed for benzene on $\mathrm{Cu}(110)$ and for benzene on the model cluster indicate that this cluster is actually too reactive and provides a poor chemical model for the system.

\section{Introduction}

The nature of the interaction of benzene with metal surfaces is of interest in various fields of applied research such as corrosion protection, lubrication, and dye adhesion as these all involve interfaces between organic matter and metals. This problem has also attracted attention in the area of heterogeneous catalysis owing to the role of metals as catalysts in ring-cracking reactions. ${ }^{1}$ Being the smallest aromatic molecule, benzene has frequently been employed as a model system for larger hydrocarbons. Recently, new interest has arisen in the interaction of aromatic compounds with metals because of their potential application in the design of devices based on electroactive organic molecules. ${ }^{2}$ For this, the prototype system is a two terminal device formed by two gold electrodes spanned by a single chemisorbed 1,4-benzenedithiol molecule. ${ }^{3}$ Thiols are known to form strong bonds of order $30 \mathrm{kcal} \mathrm{mol}^{-1}$ to gold, ${ }^{4-9}$ and to provide weaker and possibly more flexible means of attachment, the binding of aromatic azines to $\mathrm{Au}(111)$ such as pyridine and 1,10-phenanthroline have also been investigated. ${ }^{10-12}$ Always, the conformation of the molecule at the surface is critical to function; this involves both site geometry and internal 
rearrangements of the metal and adsorbate. Given the progress in theoretical chemistry combined with increasing computer power, it can be expected that computational methods will be able to reveal significant detailed information concerning these processes. A prerequisite for this, however, is to establish that computational methods give reliable predictions for each of the properties of interest, not only structural properties but also thermodynamic, spectroscopic, and process-related ones.

In previous computational studies, we have investigated the adsorption of pyridine $^{12}$ and phenylthiol ${ }^{9}$ on $\mathrm{Au}(111)$. Both of these adsorbates have end groups that anchor to $\mathrm{Au}(111)$, producing strong binding in the case of the thiol and medium-strength binding in the case of the azine. However, both adsorbates are predicted to bind over a wide range of orientational angles to the surface. For pyridine, the vertical orientation involving interactions between the nitrogen donor and the surface is predicted to be the most stable one, but flat structures dominated by $\pi$-stacking are found to be only $5 \mathrm{kcal}$ $\mathrm{mol}^{-1}$ higher in energy. For phenylthiol, little preference for either $\mathrm{sp}^{2} \mathrm{or} \mathrm{sp}^{3}$ hybridization of the sulfur is predicted, with low-energy configurations occurring for both vertically oriented and near-flat adsorbates. For both chemical systems, the nature of the intrinsic interaction between an aromatic $\pi$ system and the surface is thus quite important, and to elucidate this more directly we study herein the adsorption of benzene on $\mathrm{Au}(111)$. However, as experimental studies of this system are rare, we consider also the related systems of benzene on $\mathrm{Ag}(111), \mathrm{Cu}(111)$, and $\mathrm{Cu}(110)$ for which more information is available to characterize the effectiveness of the available computational procedures.

In an early work by Somorjai ${ }^{1 ; 13}$ it was deduced that benzene does not adsorb on either clean or stepped $\mathrm{Au}(111)$, whereas naphthalene interacts strongly with both. ${ }^{13}$ However, Wöll ${ }^{14}$ recently studied monolayers of several hydrocarbons adsorbed on various metal surfaces using X-ray absorption spectroscopy and found that benzene does indeed weakly physisorb on $\mathrm{Au}(111)$. 
The spectra indicate a high degree of molecular orientation and preserved adsorbate planarity. For benzene on $\mathrm{Ag}(111)$, a $(3 \times 3)$ ordered superstructure has been reported. ${ }^{15}$ Given the similarity in both atomic and electronic structure between gold and silver crystals, qualitative comparison of our findings for $\mathrm{C}_{6} \mathrm{H}_{6} / \mathrm{Au}(111)$ with those on $\mathrm{Ag}(111)$ can be made. In general, benzene adsorption on coinage metals takes place only below ${ }^{15 ; 16} 280 \mathrm{~K}$, indicative of the relatively weak binding. Extensive experimental data is available for the adsorption of benzene on $\mathrm{Cu}(111)$ and $\mathrm{Cu}(110)$, with the observed desorption temperature ranges being ${ }^{17 ; 18} \sim 225 \mathrm{~K}$ for $\mathrm{Cu}(111)$ and $\sim 280 \mathrm{~K}$ for the more open $\mathrm{Cu}(110)$ surface. ${ }^{19}$ Several computational studies ${ }^{20-23}$ have also considered $\mathrm{C}_{6} \mathrm{H}_{6} / \mathrm{Cu}(110)$, with variable degrees of success.

Here we report results from calculations on the adsorption of benzene on the (111) surface of $\mathrm{Cu}, \mathrm{Ag}$, and $\mathrm{Au}$ as well as on the $\mathrm{Cu}(110)$ surface. The study is carried out initially using density-functional theory (DFT), employing both atomic slab and cluster representations of the metal substrate. As significant computational problems arise for interactions like this involving very shallow potential-energy surfaces supporting very different structures with similar binding energies, a range of computational methods are investigated. In addition, we also perform second-order Møller-Plesset (MP2) perturbation-theory calculations ${ }^{24}$ based on Hartree-Fock self-consistent field (SCF) wavefunctions, as well as multi-reference complete active space (CASSCF) perturbationtheory (CASPT2) calculations. ${ }^{25}$ The results predict that the most significant contribution to the binding comes from the dispersive interaction, an interaction which at present is poorly and inconsistently accounted for by the exchange-correlation functionals used in modern applications of DFT. 


\section{Methods}

DFT computations were carried out using the packages VASP, ${ }^{26 ; 27}$ CASTEP, ${ }^{28}$ and SIESTA. ${ }^{29 ; 30}$ In the VASP and CASTEP calculations, plane-wave basis sets are employed to expand the electronic wavefunctions. Electron-ion interactions are accounted for through the use of ultrasoft pseudopotentials, ${ }^{31 ; 32}$ allowing for the use of a low energy cut-off for the plane-wave basis set. For electron-electron exchange and correlation interactions the functional of Perdew and Wang (PW91), ${ }^{33}$ a form of the generalized gradient approximation (GGA), was used in both the VASP and CASTEP calculations with an energy cut-off of the basis set set at $290 \mathrm{eV}$, as dictated by the pseudopotential for carbon. CASTEP computations were also performed using the GGA functional of Perdew, Burke, and Ernzerhof $(\mathrm{PBE})^{34}$ with the energy cut-off set to $400 \mathrm{eV}$. In the SIESTA calculations, norm-conserving pseudopotentials were used, generated according to the scheme of Troullier and Martins, ${ }^{35}$ with relativistic corrections added for the $\mathrm{Cu}$ atoms. The atomic basis set for the valence electron wavefunction expansion was of double zeta plus polarization quality. These atomic orbitals have finite range with an excitation energy of 5 mRy arising due to the confinement. Only the PBE functional was used in the SIESTA computations, while the effects of basis-set superposition error (BSSE) associated with the atomic-orbital basis set were examined using the counterpoise method. ${ }^{36}$

The surfaces of $\mathrm{Cu}, \mathrm{Ag}$, and $\mathrm{Au}$ were modeled by supercells consisting of several atomic layers and vacuum. The application of periodic boundary conditions in all three Cartesian directions yields an infinite array of periodically repeated slabs separated by regions of vacuum. A single molecule was placed in the vacuum region on the upper side of the slab. Calculations pertinent to gas-phase molecules employed a cell of the same size as the supercell of the complex, an integration using the $\Gamma$-point only, and Gaussian smearing. For 
the VASP calculations, the dipole moment arising from the asymmetric slab was compensated for by the introduction of a dipole sheet of the same strength and opposite direction in the middle of the vacuum. ${ }^{37}$ This correction can be essential for systems involving strongly dipolar or polarizing adsorbates, but has minimal affect for physisorbed benzene.

Only VASP calculations were performed for adsorbates on (111) surfaces. For these, the slabs were four atomic layers thick while the vacuum was ten. For $\mathrm{Au}$, the interlayer spacing was taken from the previously evaluated ${ }^{38}$ value of the bulk lattice parameter, $4.20 \AA$, while for $\mathrm{Ag}$ and $\mathrm{Cu}$ the corresponding values were 4.170 and $3.655 \AA$, respectively. The calculations employed $(3 \times 3)$ superstructure resulting in nine metal atoms per layer. This represents a 1/9 monolayer (ML) coverage, sufficiently low that the molecules in adjacent cells are well separated. Brillouin-zone integrations were performed using the $3 \times 3 \times 1$ k-point Monkhorst-Pack grid, with a Methfessel-Paxton smearing ${ }^{39}$ of $0.2 \mathrm{eV}$. In all computations involving the (111) slabs, the top layer and adsorbed species were allowed to relax, with other layers frozen so as to simulate a semi-infinite solid.

To keep the distance between adsorbates in neighboring cells on the $\mathrm{Cu}(110)$ lattice close to that for the $(111)$ surface, a $(2 \times 3)$ surface supercell of the original unit cell was used. This corresponds to a 1/6 ML benzene coverage. For the VASP calculations on the $\mathrm{Cu}(110)$ surface, the slab was six atomic layers thick while the vacuum was fifteen $(20 \AA)$, with the lattice parameter of $3.655 \AA$ set to match the appropriate calculated value for bulk copper. The top three layers of $\mathrm{Cu}(110)$ and adsorbed species were allowed to relax, with other layers fixed in their bulk positions. Brillouin-zone integrations were performed using the $4 \times 3 \times 1$ k-point Monkhorst-Pack grid, with a Methfessel-Paxton smearing ${ }^{39}$ of $0.2 \mathrm{eV}$. In the CASTEP and SIESTA computations, the $\mathrm{Cu}(110)$ slab was four atomic layers thick with a vacuum region of $20 \AA$. Test SIESTA calculations indicate a maximum variation in binding energy of $0.35 \mathrm{kcal} \mathrm{mol}^{-1}$ on expan- 
sion through to 7 layers. Lattice parameters of 3.636 (CASTEP) and 3.680 (SIESTA) $\AA$ for $\mathrm{Cu}$ were used consistent with the optimized values for the bulk material obtained using the PBE density functional with the appropriate basis sets. Also, $5 \times 5 \times 1$ and $3 \times 3 \times 1$ Monkhorst-Pack meshes were employed in the SIESTA and CASTEP calculations, respectively. The $\mathrm{Cu}$ slabs were fixed at their bulk geometry, however, as test calculations for the $\mathrm{Cu}(110)$ slab where the top two layers were allowed to relax, showed that this had negligible effect on binding energies.

The cluster model of Triguero et al, ${ }^{21}$ sketched elsewhere, ${ }^{22}$ was also used to study $\mathrm{C}_{6} \mathrm{H}_{6}$ on $\mathrm{Cu}(110)$. It comprises a $\mathrm{Cu}_{13}$ cluster with two atomic layers containing four atoms in the first layer that are bonded to a benzene molecule. The cluster has overall $\mathrm{C}_{2 v}$ symmetry; strong chemisorptive-type interactions distort the benzene ring, however, giving it an inverted boat shape akin to the quinonoid form of the lowest excited triplet state ${ }^{40}$ of the benzene in the gas phase. $^{21}$

The CASPT2 calculations $^{25}$ were performed using the MOLCAS package. ${ }^{41}$ The Stuttgart basis set ECP10MWB ${ }^{42}$ with its $1 \mathrm{~s}+2 \mathrm{~s}+2 \mathrm{p}$ effective core potential was used for $\mathrm{Cu}$ in conjunction with the $6-31+\mathrm{G}^{*}$ basis set ${ }^{43}$ for $\mathrm{C}$ and $\mathrm{H}$. The active space was chosen in a way that would comprise all $13 \mathrm{Cu} 4 \mathrm{~s}$ electrons distributed through all $13 \mathrm{Cu} 4 s$ orbitals. The chosen orbitals were $5 \mathrm{a}_{1}, 2 \mathrm{a}_{2}, 3 \mathrm{~b}_{1}$, and $3 \mathrm{~b}_{2}$ while the doubly occupied orbitals were $41 \mathrm{a}_{1}, 28 \mathrm{a}_{2}, 34 \mathrm{~b}_{1}$, and $35 \mathrm{~b}_{2}$. Orbital rotations distorted this picture, however, with some benzene occupied orbitals, benzene virtual orbitals, copper $3 d$ occupied orbitals, and copper $4 p$ virtual orbitals being swapped into the active space instead of some of the copper $4 s$ orbitals. One $\mathrm{Cu} 3 p$ orbital was also occasionally rotated into the active space. To eliminate the effects of this rotation, the complete-activespace self-consistent-field (CASSCF) part of the CASPT2 calculations were also performed using a frozen core consisting of the $\mathrm{C} 1 s$ and $\mathrm{Cu} 3 s+3 p$ orbitals. The quantitative effects of this restriction were insubstantial, however, 
and the results are not presented. In both cases the Møller-Plesset perturbation aspect of the CASPT2 calculations was performed using frozen $\mathrm{C} 1 s$ and $\mathrm{Cu} 3 s+3 p$ orbitals. In addition to the CASPT2 calculations, second-order Møller-Plesset perturbation (MP2) ${ }^{24}$ calculations were also performed based on a two-determinant restricted open-shell Hartree-Fock (ROHF) wavefunction using the GAUSSIAN03 program package ${ }^{44}$ with the same basis sets and frozen orbitals. All binding energies were corrected for basis-set superposition error using the counterpoise method. ${ }^{36}$ Also, some constrained optimizations of the geometry of the adsorbate above the $\mathrm{Cu}_{13}$ cluster were performed by GAUSSIAN03 using the PW91 density functional ${ }^{33}$ with the ECP10MWB ${ }^{42}$ and $6-31 \mathrm{G}^{*}$ basis sets, but without use of BSSE correction.

\section{Results and Discussion}

\subsection{VASP PW91 calculations of adsorption in the low-coverage limit}

The adsorption of benzene on the $\mathrm{Cu}(111), \mathrm{Ag}(111)$, and $\mathrm{Au}(111)$ surfaces is considered for flat-lying orientations in which the center of the ring is classified as being either above TOP, bridge (BR), or FCC/HCP three-fold hollow sites on the surface. Six high-symmetry binding configurations are illustrated in Fig. 1 for which the corresponding adsorption energy changes $\Delta E$, evaluated using VASP, are listed in Table 1. These comprise two orientations each, named A and B, for binding at the four sites. All optimized coordinates are provided in Supporting Information.

For benzene on $\mathrm{Cu}(111)$, the two TOP sites are calculated to provide no binding at all, while the BR, FCC, and HCP sites support only very weak binding of $\Delta E \sim-0.5 \mathrm{kcal} \mathrm{mol}^{-1}$. This result is in stark contrast with the observed binding of $\Delta E=-14 \mathrm{kcal} \mathrm{mol}^{-1}(-0.6 \mathrm{eV}) .{ }^{17 ; 18}$ 
For the case of benzene on $\operatorname{Ag}(111)$, the calculated interaction energies for the 8 structures are all more attractive by $0.1-1.0 \mathrm{kcal} \mathrm{mol}^{-1}$ than the corresponding values for benzene on $\mathrm{Cu}(111)$. No experimental value for the binding energy is available, but chemical arguments ${ }^{45}$ suggest that instead it should $n o t$ be as strongly bound. However, the strongest interaction of $\Delta E \sim-1.2 \mathrm{kcal}$ $\mathrm{mol}^{-1}$ on $\mathrm{Ag}(111)$ is predicted for the FCC hollow site in the orientation " $\mathrm{A}$ " in a pattern that actually corresponds to the experimentally observed $(3 \times 3)$ superstructure found after an exposure to $5 \mathrm{~L}$ of benzene on $\operatorname{Ag}(111) .{ }^{15} \mathrm{An}$ early molecular-orbital calculation also suggested that a three-fold hollow site is most favored for benzene adsorption on a silver cluster. ${ }^{46}$ The most significant conclusions to be drawn from the calculations, however, is that benzene is predicted to wander freely across the surface with little barrier, even at low temperatures.

The VASP calculations predict also that for each possible binding site the interaction energy is 0.6 - $0.8 \mathrm{kcal} \mathrm{mol}^{-1}$ more favourable for binding to $\mathrm{Au}(111)$ than to $\mathrm{Ag}(111)$. Binding to the HCP hollow site A is only $0.1 \mathrm{kcal} \mathrm{mol}^{-1}$ more favourable than the FCC hollow site, however, again indicating no significant preference for any particular binding site.

The adsorption of benzene on $\mathrm{Cu}(110)$ is considered at four different binding sites, as illustrated in Fig. 2, termed the TOP, hollow (HOL), short bridge (SB) and long bridge (LB) sites. On each site two high symmetry orientations of the molecule are considered, and these are named A and B in the figure. In addition, three possible adsorbate structures are also considered corresponding to physisorption of flat molecules and possible chemisorption involving quinonoid and $\mathrm{H}$-flipped configurations of the ring; ${ }^{21 ; 22}$ these structures are illustrated in Fig. 3. The chemisorbed structures correspond directly to the local minima identified ${ }^{21 ; 22}$ in calculations of benzene above the HOL site of a $\mathrm{Cu}_{13}$ cluster used as a model for the (110) surface. The computed binding energies are given in Table 2 while key structural properties are given in Table 
3 and all optimized coordinates are provided in Supporting Information. Some of these key properties include the average height of the carbon atoms above the surface, $\Delta z$, the maximum difference in CC bond lengths, $\Delta R_{C C}$, and the maximum $\mathrm{CCCC}$ and $\mathrm{CCCH}$ torsional angles, $\tau_{C C C C}$ and $\tau_{C C C H}$, respectively. Based upon them, the optimized structures are classified as being either "flat" $\left(\Delta z=2.7-2.9 \AA, \Delta R_{C C}<0.006 \AA\right.$, and torsional angles $<2.5 \mathrm{deg}$ in magnitude), "quinonoid" $\left(\Delta z=2.0-2.4 \AA, \Delta R_{C C}\right.$ up to $0.06 \AA$, large positive $\tau_{C C C C}$ and large positive $\left.\tau_{C C C H}\right)$, and "H- flipped" $\left(\Delta z=2.0-2.4 \AA, \Delta R_{C C}\right.$ up to $0.02 \AA$, significant negative $\tau_{C C C C}$ and large positive $\left.\tau_{C C C H}\right)$. In some cases, geometry optimization leads to local-minimum structures with qualitative properties preserved, while for the remainder the structures relaxed to an alternate configuration, as indicated in Table 2.

¿From the results in Table 2, an important qualitative feature is that increased binding by ca. $5 \mathrm{kcal} \mathrm{mol}^{-1}$ is predicted for benzene binding to $\mathrm{Cu}(110)$ compared to $\mathrm{Cu}(111)$. While this is consistent with the observed increase of 9 kcal $\mathrm{mol}^{-1},{ }^{17 ; 18 ; 23}$ the absolute magnitude of the binding energies remain in poor agreement, $6 \mathrm{kcal} \mathrm{mol}^{-1}$ calculated compared to $23 \mathrm{kcal} \mathrm{mol}^{-1}$ observed. ${ }^{23} \mathrm{Ph}$ ysisorbed structures are predicted to be more stable than chemisorbed ones, but the energy difference is only $0.6 \mathrm{kcal} \mathrm{mol}^{-1}$, a value that is most likely less in magnitude than the accuracy of the methodology. As Table 3 shows that these local minima differ dramatically in structure, and as qualitatively we find no significant barriers separating them, it is clear that very large amplitude motions may be sustainable on the surface and hence proper quantum thermal treatment of the vibrational motion will be essential in any quantitative comparison of computed and experimental properties for the system. However, STM images of benzene on $\mathrm{Cu}(110)$ have revealed that the adsorbates stick over both the long-bridge site $^{47}$ and the hollow site, ${ }^{48}$ the two lowest- energy sites revealed in Table 2 . Also, it has been observed ${ }^{47}$ that benzene is easily dragged over $\mathrm{Cu}(110)$ by an STM tip; this is consistent with the 
basic qualitative scenario predicted by the calculations of poorly site-specific binding.

Significant differences are found between the optimized structures of benzene on $\mathrm{Cu}(110)$ and those reported previously by Triguero et al. ${ }^{47 ; 48}$ for the binding of benzene to a $\mathrm{Cu}_{13}$ cluster. Shown in Table 3 are the geometrical parameters from these calculations for both the cluster-optimized HOL-2A (quinonoid) and HOL-2B (H-flipped) structures, as well as those for our corresponding surface-optimized structures. On the surface, the distortion to the benzene geometry is dramatically reduced, and the molecule floats ca. $0.2 \AA$ higher above the surface. The calculated interaction energies are also very different, with those for the cluster being -18 and $-14 \mathrm{kcal} \mathrm{mol}^{-1}$ for the quinonoid and H-flipped structures, respectively, compared to $-2.9 \mathrm{kcal} \mathrm{mol}^{-1}$ and $-5.2 \mathrm{kcal}$ $\mathrm{mol}^{-1}$, respectively, on the surface. Further, no flat structures are found above the cluster whereas a flat structure forms the most stable structure, of interaction energy $-5.8 \mathrm{kcal} \mathrm{mol}^{-1}$, on the surface. As there are some computational differences between the original DFT implementation and that used herein, we repeated the previous cluster calculations using VASP and PW91 for the HOL-A quinonoid structure, obtaining $\Delta E=-19 \mathrm{kcal} \mathrm{mol}^{-1}$ in excellent agreement with the previous value. Hence the differences are due primarily to the differing reactivities of the cluster and the surface. The reasonable agreement found prevously between the cluster binding energy and the surface observed adsorption energy is thus found to be due to the near cancellation of two significant effects: the underestimation of the binding due to limitations associated with modern DFT functionals, and the enhanced reactivity of the cluster. Note also that variation of the DFT functional used does not lead to qualitative changes in the results, and that the problems encountered with the calculation by DFT of the binding of benzene to coinage-metal surfaces is general.

The adsorption of benzene on $\mathrm{Cu}(111)$ is known ${ }^{14 ; 15 ; 17}$ to be physisorptive 
in nature. A series of calculations has been performed to determine whether or not VASP predicts stable chemisorbed species for this surface, as it does for $\mathrm{Cu}(110)$. Geometry optimizations were performed starting at analogous quinonoid and H-flipped conformations. In all cases, the geometries relaxed to the flat ones, indicating that the surface calculations do not intrinsically overestimate the significance of the chemisorbed structures, and hence they remain as viable alternatives for the actual structure on $\mathrm{Cu}(110)$. We return to the question of the experimental determination of whether the interaction is fundamentally chemisorptive or physisorptive in Section 3.3.

\subsection{Verification of the major results using CASTEP and SIESTA calcula- tions}

The VASP calculations reveal potential-energy surfaces that support $0.6 \AA$ changes in the metal-adsorbate separation $\Delta z$ and large intramolecular distortions $\Delta R_{C C}, \tau_{C C C C}$ and $\tau_{C C C H}$ to the adsorbate at the cost of the very small amount of ca. $1 \mathrm{kcal} \mathrm{mol}^{-1}$ in energy. Such energy changes are less than absolute error magnitudes expected for modern density-functionals, pseudopotentials and basis sets, while the determination of precise results is computationally challenging in terms of the algorithms used for geometry optimization, etc.. To verify that the major conclusions reached from the VASP calculations are robust to these effects, some analogous calculations have been performed using CASTEP and SIESTA for the binding of benzene to the surface of greatest contention, the $\mathrm{Cu}(110)$ surface.

The CASTEP calculations were performed for the LB-A and LB-B structures involving translational scans of the potential-energy surface for frozen metal and adsorbate components, using the flat, quinonoid, and H-flipped adsorbate structures. In all cases, the same qualitative conclusions were reached as from the VASP optimizations. For one structure a full optimization was performed 
and this yielded a binding energy within $1 \mathrm{kcal} \mathrm{mol}^{-1}$ of the corresponding VASP one. For most problems such quantitative agreement would be considered excellent, but for this system this amounts to $20 \%$ of the binding energy. The significant factor, however, is that the primary qualitative conclusions remain invariant. CASTEP was also used to compare results from the PW91 and PBE density functionals; good agreement was found, with the PBE binding strengths being slightly less than the PW91 values by just $0.1-0.3$ kcal $\mathrm{mol}^{-1}$.

SIESTA calculations were performed for all adsorbate structures and binding locations, and the results are provided along with the VASP ones in Table 2. These calculations were performed by first adjusting the height of the adsorbate above the surface at fixed metal and adsorbate geometry so as to provide a best-estimate starting structure, and then these structures were fully relaxed. Both the energy of the $z$-optimized structure and the fully relaxed one are given in the table. The resultant quinonoid and H-flipped structures show even less variations in bond lengths and torsional angles than those from the VASP calculations reported in Table 3, with in particular the HOL-A structure being very flat; significant differences in the height above the surface are still found between the physisorbed and chemisorbed structures, however.

Direct comparison of the binding energies from the SIESTA and VASP calculations is difficult owing to the significant BSSE that arises from the use of atomic basis sets in the SIESTA calculations. While atomic basis sets are much more conducive to mechanistic analyses than are plane-wave ones, an advantage exploited in the next subsection, the presence of BSSE arising from the incompleteness of the atomic basis set used provides a significant disadvantage. In Table 2, the energy changes due to binding are shown both with and without the use of BSSE corrections. The energies of binding without BSSE correction fall in the range of $\Delta E=-7$ to $-20 \mathrm{kcal} \mathrm{mol}^{-1}$ but after correction the binding is or is very nearly lost altogether. The corresponding 
values obtained using VASP and CASTEP fall mid-way between the BSSE corrected and uncorrected values. When large atomic basis sets are used, the BSSE correction is usually small and typically of the wrong sign and so BSSE corrections should not be applied. ${ }^{49}$ However, for small atomic basis sets, the BSSE correction is large and of the correct sign and its application is essential. The double-zeta plus polarization basis set used in these SIESTA calculations does not have the augmented functions that are crucial to BSSE reduction and hence its application appears essential. However, for intermediate-sized basis sets such as this, a technique of fractional BSSE correction is often used ${ }^{50}$ involving the addition of some set fraction of the full correction. This technique may be applicable here, with fractional corrections of 0.6 - 0.9 being required to bring the SIESTA and VASP results into quantitative agreement.

A significant difficulty with the atomic basis set approach, however, is that all geometry optimizations are performed on the raw, uncorrected energies. As the BSSE is of order $6 \mathrm{kcal} \mathrm{mol}^{-1}$ for the distant physisorbed structures and of order $14 \mathrm{kcal} \mathrm{mol}^{-1}$ for the close-lying chemisorbed ones, the method used to treat it induces significant changes to the shape of the potential-energy surfaces. As a result, e.g., the raw SIESTA energies for the HOL-A structure strongly favour the H-flipped structure whilst after correction they favour the flat one. As the reduction of BSSE to the level required for realistic geometry optimization in these systems requires Gaussian basis sets that are at least an order of magnitude larger than those used herein, ${ }^{49}$ any previous or foreseeable calculation of this type, is likely to be unreliable. Such calculations will artificially favour closely interacting, highly distorted chemisorbed structures over physisorbed ones. While this effect cannot account for the perceived high reactivity of the $\mathrm{C}_{13}$ cluster to benzene, it could account for the high degree of distortion found in the cluster-optimized structures. 


\subsection{The electronic structure of the adsorbate layer}

Adsorption-induced changes to the electronic structure of the surface and adsorbate provide important indicators of the nature of the surface - molecule interaction. Atomic-basis-set programs such as SIESTA provide insight into this process through the ready application of simple methods such as Mulliken analysis of the charge flow to the adsorbate, methods not available for use with plane-wave basis sets. SIESTA results for benzene on the (111) surfaces of $\mathrm{Cu}$, $\mathrm{Ag}$, and $\mathrm{Au}$ indicate negligible charge transfer to benzene, $q_{m o l}$, of magnitude less than $0.01 e$, where $e$ is the magnitude of the charge on the electron. For benzene in various structures above $\mathrm{Cu}(110)$, SIESTA results are given in Table 3; again, negligible charge transfer is found for the flat physisorbed structures, but some charge flow up to $0.12 e$ is predicted for the chemisorbed ones, especially those at the cluster-optimized geometries.

A commonly used method to estimate charge flow using plane-wave based calculations is Helmholz anaylsis of the change in the surface work function. A reduction in the work function of the surface of $-0.44 \mathrm{eV}$ is predicted by the VASP calculations for the $\mathrm{C}_{6} \mathrm{H}_{6}-(3 \times 3)-\mathrm{Ag}(111)$ surface, in reasonable agreement with the measured value of $-0.3 \mathrm{eV} \cdot{ }^{15 ; 51} \mathrm{On} \mathrm{Au}(111)$ the computed value is $-0.42 \mathrm{eV}$, while on $\mathrm{Cu}$ it is somewhat bigger: $-0.55 \mathrm{eV}$ (obs. ${ }^{52}-0.3 \mathrm{eV}$ ) and $-0.84 \mathrm{eV}$ on the $(3 \times 3)-(111)$ and $(2 \times 3)-(110)$ surfaces, respectively. Using the Helmholz equation ${ }^{1}$ applied in the low coverage limit, charge transfer to the adsorbate may be estimated from these changes in surface potential, yielding $0.71,0.74,0.81$, and $1.26 \mathrm{D}$ per adsorbate molecule for the calculated lowestenergy structures on $\mathrm{Au}(111), \mathrm{Ag}(111), \mathrm{Cu}(111)$, and $\mathrm{Cu}(110)$, respectively. Dipole-moment changes arise from polarization of the metal surface, polarization of the molecule, and from charge-transfer between the molecule and surface. Neglecting polarization effects completely leads to estimated charge transfers of $0.04,0.04,0.05$, and $0.10 e$ for these flat adsorbates, much larger 
than the values of $<0.01 e$ deduced from the SIESTA Mulliken analysis. This discrepancy could arise as the molecular and surface polarization terms are also naively expected to be of this order but of opposite sign to each other. Also, non-Helmholz terms do contribute to changes in the surface potential, ${ }^{53-55}$ and such effects could dominate the process especially for weakly bound adsorbates.

The predicted and observed changes in the work function are much smaller than those predicted and observed for benzene chemisorbed on reactive transition metals such as $\mathrm{Ni}, \mathrm{Pd}$, and Pt of ca. $1.4 \mathrm{eV} .{ }^{1}$ There is thus a significant qualitative difference found between the results of the present calculations and those for a system in which full organometallic bonds are implied. Analysis of the work function changes calculated from the plane-wave-based methods thus corroborates the conclusions reached from Mulliken analysis of the SIESTA results that DFT predicts only weak to very weak interactions between benzene and the various surfaces.

To gain further insight from the VASP calculations into the electronic variations that arise because of adsorption, projected densities of states (PDOS) have been evaluated. Results are presented in Figures 3 and 4 for benzene and $\mathrm{Cu}(110)$ well separated, at the optimized flat HOL-B lowest-energy structure, and for the starting (ie., $\mathrm{C}_{13}$ cluster optimized ${ }^{21 ; 22}$ ) and surface-optimized HOL-A quinonoid and HOL-B H-flipped structures. Fig. 3 shows the average $\mathrm{C}$ $p_{z}(\pi)$ orbital density while Fig. 4 shows the density for the surface copper $d_{z^{2}}$ orbitals. As it has been shown that the computational methods predict much more realistic changes in binding energies between the $\mathrm{Cu}(111)$ and $\mathrm{Cu}(110)$ surfaces than absolute binding energies, results for benzene on $\mathrm{Cu}(111)$ at the lowest-energy optimized HCP-hollow A are also included in these figures. In addition, results from the quantitative analysis of the calculated PDOS for all cluster and surface optimized structures are provided in Table 3. These include the highest-occupied molecular orbital (HOMO) energy with respect 
to the Fermi level $\epsilon_{H}-E_{F}$, the shifts of the lowest-unoccupied molecular orbital (LUMO) from the gas phase, $\epsilon_{L}^{g a s}$, as well as from its calculated value on $\mathrm{Cu}(111), \epsilon_{L}^{111}$.

The gas-phase densities show the benzene HOMO at $2.15 \mathrm{eV}$ below the Fermi energy $E_{F}$, whereas this band is observed ${ }^{56}$ to be at $4.5 \mathrm{eV}$ below the $\mathrm{Cu}(110)$ Fermi energy (this Fermi energy is at $-4.8 \mathrm{eV}$ with respect to the vacuum level); alternatively, the calculated LUMO appears at $2.96 \mathrm{eV}$ above $E_{F}$ compared to $5.9 \mathrm{eV}$ observed. These discrepancies are due to the asymptotic potential error and band-gap error, respectively, that are inherent in modern density functionals; $;^{9 ; 57}$ the calculated band gap is $5.1 \mathrm{eV}$ while the observed one is $10.3 \mathrm{eV}$. The HOMO orbital error is somewhat compensated for in calculations of surface adsorbates by charge transfer processes that act to align the energy level systems. As a result, DFT calculations tend to give qualitatively reasonable occupied electronic structures of adsorbates but fail to quantitatively reproduce charge transfer. ${ }^{9}$

For benzene on $\mathrm{Cu}(111)$, Fig. 4 shows that the calculated $\mathrm{C} \pi$ PDOS is broadened slightly due to the weak interaction with the metal surface and shifted downward by $0.66 \mathrm{eV}(\mathrm{HOMO})$ and $0.40 \mathrm{eV}$ (LUMO). These shifts reflect the net effects of orbital-specific molecule-surface interactions and charge transfer. More significant interactions are evident for benzene on $\mathrm{Cu}(110)$, however. For the deduced lowest-energy physisorbed flat structure HOL-B, the calculated $\mathrm{C} \pi$ PDOS shown in Fig. 4 is broadened significantly, shifted downward, and split into two peaks at 1.36 and $1.77 \mathrm{eV}$, changes $\Delta \epsilon_{L}^{g a s}$ given in Table 3 of -1.57 and $-1.17 \mathrm{eV}$ from the gas-phase values. The corresponding HOMO level shifts down by $1.37 \mathrm{eV}$. For the optimized chemisorbed structures, the broadening of the LUMO (and HOMO) is further increased while the orbitals shift to lower energy as the separation $\Delta z$ decreases and interactions become significantly larger. However, the PDOS evaluated for the quinonoid and $\mathrm{H}-$ flipped geometries optimized in previous cluster calculations ${ }^{21 ; 22}$ show even 
greater broadening and shifts, with the LUMOs shifted until they cross the Fermi energy. The SIESTA Mulliken charge analysis results shown in Table 3 also indicate the appearance of detectable charge transfer in these chemisorbed structures, up to $0.12 e$ at the cluster-optimized geometries. Note that a possible consequence of the DFT band-gap error is that the charge transfer process associated with the donation of electrons from the benzene $\pi$ orbitals to the metal is artificially curtailed by the apparent back-bonding that is enforced when the LUMO prematurely crosses the metal Fermi energy.

Qualitatively, the interaction of benzene with $\mathrm{Cu}(110)$ is known to be much weaker than that with $\mathrm{Ni}(100),{ }^{58}$ a surface on which it is clearly chemisorbed. Also, the benzene - $\mathrm{Cu}(110)$ interaction is much weaker than that of acetylene with $\mathrm{Cu}(110),{ }^{59}$ another chemisorptive interaction, but it is significantly stronger than the interaction of benzene and $\mathrm{Cu}(111)$, a clearly physisorptive interaction. ${ }^{14 ; 15 ; 17}$ The adsorption of benzene on $\operatorname{Ag}(111)$ is also unambiguously physisorptive. ${ }^{15 ; 51}$ While some experimental results ${ }^{58}$ have been interpreted in terms of weak physisorption of benzene on $\mathrm{Cu}(110)$, others ${ }^{21 ; 22 ; 60}$ have been interpreted as indicating that $\sigma-\pi$ mixing does occur and hence some degree of chemisorption is implicated. Indeed, the DOS for all of the possible optimized structures shown in Fig. 4 or summarized in Table 3 depict significant interactions between benzene and $\mathrm{Cu}(110)$, interactions that are much stronger than those with $\mathrm{Cu}(111)$, in agreement with the general scenario depicted experimentally. PW91 calculations ${ }^{61}$ for benzene on $\mathrm{Ni}(110)$ predict a binding energy of $41 \mathrm{kcal} \mathrm{mol}^{-1}$ and torsional angles up to $35^{0}$, clearly depicting strong chemisorption as apposed to the much weaker binding on $\mathrm{Cu}(110)$, also in agreement with experimental findings.

More quantitative experimental information is available, however, that could in principle discriminate between the various calculated binding possibilities. X-ray emission spectroscopy observes the nature of the occupied orbitals. For benzene on $\mathrm{Ni}(110)$, the $\mathrm{HOMO}$ orbital is observed at an energy of -4.3 to 
$-4.6 \mathrm{eV}$ with respect to the Fermi level and calculated in good agreement by PW91 to be at $-4.5 \mathrm{eV} \cdot{ }^{61}$ For benzene on $\mathrm{Cu}(110)$, the observed value ${ }^{58}$ is very similar, $-4.4 \mathrm{eV}$. Figure 4 shows that the calculated HOMO levels are of this order but vary considerably depending on site and structure. In Table 3, the calculated HOMO energies $\Delta \epsilon_{H}-E_{F}$ are listed. Most calculated structures predict $\Delta \epsilon_{H}$ within $0.4 \mathrm{eV}$ of the observed value, the exceptions being the cluster-optimized structures, at ca. $-7 \mathrm{eV}$, and the high-energy TOPA structures at $>-3 \mathrm{eV}$. The best results are obtained for the optimized quinonoid LB-A and H-flipped HOL-B and LB-B structures $(-4.0$ to $-4.3 \mathrm{eV})$ while the low-energy flat structures all more significantly removed (-3.4 to -3.5 $\mathrm{eV})$.

The energy difference between the LUMO orbital and the Fermi energy cannot be reliably determined using modern DFT owing to the DFT band-gap error, but changes in this quantity between different structures should be better described. The calculated changes $\Delta \epsilon_{L}^{111}$ between adsorbates on $\mathrm{Cu}(110)$ and $\mathrm{Cu}(111)$ given in Table 3 and are -1.2 and $-0.8 \mathrm{eV}$ for the two peaks associated with the lowest-energy surface optimized (HOL-B) structure. These are somewhat less for the other physisorbed structures, $-1.7 \mathrm{eV}$ and $-2.2 \mathrm{eV}$ for the cluster-optimized quinonoid (HOL-A) and H-flipped (HOL-B) structures, respectively, and -1.0 to $-1.3 \mathrm{eV}$ for surface optimized quinonoid and H-flipped structures. Experimentally the LUMO energy for benzene on $\mathrm{Cu}(111)$ has been determined from inverse photoemission spectroscopy. ${ }^{62}$ Figure 6 shows the original results ${ }^{62}$ fitted to Gaussian-shaped peaks on a piecewise-linear background. The inverse photoemission spectrum for clean $\mathrm{Cu}(111)$ is also shown; it contains two peaks that are lost in the absorbate but more significantly a very similar underlying background. For benzene on $\mathrm{Cu}(111)$, the fitted Gaussian has a centre of $4.4 \mathrm{eV}$ and standard deviation of $0.8 \mathrm{eV}$. The LUMO energy for benzene on $\mathrm{Cu}(110)$ has been measured by scanning-tunnelling spectroscopy ${ }^{48}$ and the original current-voltage $(I(V))$ curve is shown in Figure 7 . There the 
curve is fitted to the sum of three arctan functions depicting molecular resonances $^{63}$ at $3.0 \mathrm{eV}$ (LUMO), $3.7 \mathrm{eV}$, and $4.5 \mathrm{eV}$. Hence the observed value of $\Delta \epsilon_{L}^{111}$ is $-1.4 \mathrm{eV}$, in best agreement with the calculated results for the optimized chemisorption structures, again, although the flat HOL-B structure is quite close and actually has the two-peaked structure found experimentally.

Hence, from consideration of the PDOS, it is clear that the cluster-optimized structures depict unreasonable possibilities, while the optimized chemisoprtion structures are most favoured and the flat HOL-B structure is not implausible. Authoritative conclusions cannot be made, however, due to the complex nature of the calculated PDOS structures and the lack of treatment of quantummechanical and thermal vibrational effects. Given the small energy differences predicted between the most probable chemisorptive and physisorptive structures, it could be that zero-point or thermal vibrational effects are sufficient to mix all of these structures, resulting in an observed average structure that could be quite different in appearance to any of the local-energy minima found on the potential-energy surface.

In general, only small perturbations to the DOS of the copper atoms are found on adsorption of benzene. The copper orbital that interacts most significantly is the $d_{z^{2}}$ orbital whose PDOS is shown in Fig. 5. While a weak tail to this distribution above the Fermi energy is found indicating bonding interactions with the unoccupied molecular orbitals, the effect is clearly quite weak. Instead, large downward shifts of the orbital energies are found, especially for the cluster-optimized chemisorptive structures, indicating a strong interaction with the occupied orbitals. It is indicative of a strong dispersive interaction between the copper and benzene, an interaction for which DFT does not correctly include the resultant attractive energy contribution, especially for complexes with coinage metals. ${ }^{38 ; 49}$ It is hence reasonable to hypothesise that the binding is dispersive in nature and that this is the cause of the poor agreement between calculated and observed absolute binding energies. 


\subsection{Lack of involvement of the triplet states of benzene in the binding}

The chemisorptive interactions observed between some alkenes and reactive metal surfaces cannot be accounted for assuming that the surface interacts with the ground state of the alkene. ${ }^{21}$ Instead, strong interactions with excited states of alkenes have been invoked. For benzene on $\mathrm{Cu}(110)$, the local structure of the benzene is reminiscent of the equilibrium geometry ${ }^{40}$ of the lowest-triplet excited state, and hence it has been postulated that it is this state that interacts with the metal. Assuming that two covalent bonds form in this chemisoprtive process, ${ }^{21}$ the DFT-calculated absorption energy of 18 kcal mol${ }^{-1}$ for benzene on the $\mathrm{Cu}_{13}$ cluster has been interpreted as indicating a $\mathrm{Cu}-\mathrm{C}$ bond strength of $58 \mathrm{kcal} \mathrm{mol}^{-1}$, opposed by the energy of $98 \mathrm{kcal}$ $\mathrm{mol}^{-1}$ required to form the triplet state. Triguero et al. ${ }^{21}$ have depicted the generic form of the potential-energy surfaces expected in this situation and these are sketched in Figure 8: the ground-state of the adsorbate correlates to the asymptotic benzene triplet state while the asymptotic ground state becomes an excited state of the adsorbate. Note that both depicted states are actually doublets, the upper one being a triplet-coupled state known as a tripdoublet state. ${ }^{64 ; 65}$ In this figure is also shown the actual potential-energy surfaces for these two states calculated using DFT at the PW91/6-31G* level using GAUSSIAN03. These surfaces were obtained by freezing the cluster geometry and the height of the nearest carbon atoms above the surface, allowing all other coordinates of the benzene molecule to relax on the ground state. The ground state of the adsorbate is clearly seen to correlate to the asymptotic ground state, in contrast to the predictions of the triplet-interaction model. Note that the calculated binding energy of $24 \mathrm{kcal} \mathrm{mol}^{-1}$ is somewhat greater than the value of $19 \mathrm{kcal} \mathrm{mol}^{-1}$ obtained in this work previously using VASP; this is due largely to the neglect of BSSE corrections in the current calculations. 
Accurate calculations of the energy of the benzene tripdoublet state are not feasible as there are many hundreds of excited states of lower energy involving the cluster; at the equilibrium geometry, we determined the nature of the lowest 100 excited states using time-dependent DFT, finding none to contain benzene triplet character. However, a crude estimate of the tripdoublet energy is obtained as the difference in the benzene LUMO and HOMO orbital energies, and this is shown in the figure. Though only approximate and not optimized for the electronic state of the cluster, these results support the emphatic results obtained for the ground-state surface that the adhesion of benzene to the $\mathrm{C}_{13}$ cluster does not involve covalent bonding to the triplet state. Instead, the DFT results depict a weak intermolecular interaction typical of hydrogen bonding, dispersive interactions, or possibly dative covalent bonding involving the benzene ground state only.

Inspection of the form of the molecular orbitals reflects the same scenarios discussed in the previous section for the surface-benzene interaction: all benzene $\pi$ orbitals of the adsorbate are significantly depressed in energy, with the dominant mixings being between different occupied levels (or between different virtual levels) arising from dispersive intermolecular interactions. However, there are also some significant interactions evident between the benzene HOMO and unoccupied cluster orbitals. This results in significant charge transfer, the net effect of which is, after BSSE correction, a Mulliken-charge transfer of $q_{m o l}=$ $0.3 \mathrm{e}$; this could indicate the action of dative covalent bonding. The depression of the benzene LUMO orbital appears to be dominated by interactions with copper $4 p$ orbitals. If this interaction was slightly stronger, the LUMO could become significantly occupied and hence the benzene would appear to take on triplet character. Hence the triplet interaction model, whilst being shown to be inappropriate for benzene on $\mathrm{Cu}(110)$, may be quite apt for systems with slightly stronger interactions. 


\subsection{Quantification of the contribution of dispersion to the binding energy}

While DFT calculatons indicate that the $\mathrm{Cu}_{13}$ cluster introduced by Triguero et al. ${ }^{47 ; 48}$ is too reactive for quantitative modelling of the reactivity of $\mathrm{Cu}(110)$ surfaces, it can provide a useful guide as to the significance of dative bonding and dispersive force as it facilitates the application of high-end $a b i n i$ tio approaches designed for discrete molecular systems. We have performed CASPT2 calculations for the $\mathrm{Cu}_{13}$ - benzene interaction for the quinonoid structure HOL-A at the previous cluster-optimized geometry ${ }^{21}$ and compared them to DFT calculations for the same system. CASPT2 is a Møller-Plesset perturbation method similar to MP2 but generalized to treat systems with open-shell bands such as the $s$ band of the copper cluster and is, in principle, the simplest $a b$ initio method that is appropriate for problems of this type. It can provide an a priori estimate of the magnitude of the dispersive interaction that acts in parallel to, and independent of, covalent-bonding forces; it has been shown to be reliable for the study of related problems involving a small number of metal atoms, ${ }^{49 ; 66}$ but it becomes much more difficult to apply to large metal clusters such as $\mathrm{Cu}_{13}$.

At the cluster optimized binding geometry of Triguero et al. ${ }^{21}$ the raw interaction energy of the two fragments is calculated using CASPT2 to be -77 kcal mol ${ }^{-1}$, reducing to $-51 \mathrm{kcal} \mathrm{mol}^{-1}$ after BSSE correction. The distortion energy of the benzene molecule required to produce the quinonoid geometry is calculated at this level to be $18 \mathrm{kcal} \mathrm{mol}^{-1}$, so the total calculated interaction energy is $-33 \mathrm{kcal} \mathrm{mol}^{-1}$. The CASSCF calculations used as a starting point for the perturbation calculations in CASPT2 predict that the cluster is highly unbound, however: the raw interaction energy is $+17 \mathrm{kcal} \mathrm{mol}^{-1}$, becomes +20 kcal mol ${ }^{-1}$ after BSSE correction and gives the total interaction energy as +38 $\mathrm{kcal} \mathrm{mol}^{-1}$. As covalent bonding is described at a usefully realistic level at the CASSCF level and no binding is predicted, it is clear that covalent bonding 
plays an insignificant role in the interaction. This includes both simple dative bonding in which say benzene acts as an electron donor to fill partially occupied copper orbitals, as well as more sophistocated scenarios such as the interaction of the surface with benzene excited states. The correlation-energy correction is thus a massive $-71 \mathrm{kcal} \mathrm{mol}^{-1}$, a correction that is non-covalent in origin.

The correlation energy of two interacting species can be separated into contributions from the changes to the fluctuations on each species as modified by the presence of the other, as well as the dispersive contribution that arises from correlated fluctuations on both species. If a covalent bond forms between the two species, then the bond formation alters the valence electrons on each, and these electrons then interact with the local cores. This gives rise to the non-dispersive core - valence correlation ${ }^{67}$ that can act to significantly deepen covalent wells, ${ }^{67}$ being especially significant for interactions with transition metals. ${ }^{68}$ Core-valence correlation acts in response to bonding interactions but does not constitute a bonding mechanism. In the present application, there is no intrinsic covalent bond for core-valence correlation to enhance, but there is clear evidence of many dispersive bonding interactions that mixing say the copper $3 d$ orbitals with the benzene occupied orbitals. It is thus clear that the primary source of the binding is dispersive in nature, and that the effect of any core-valence correlation is thus to enhance the significance of the dispersive interactions.

The thirteen-orbital active space optimized in the CASSCF calculations excluded some of the $\mathrm{Cu} 4 s$ orbitals that initially constituted it, including in their stead some occupied $\mathrm{Cu} 3 d$ and benzene orbitals as well as some unoccupied $\mathrm{Cu} 4 p$ and benzene orbitals. Like results obtained using DFT, the orbital coefficients reflect strong mixing of the occupied benzene and $\mathrm{Cu} 3 d$ orbitals, and strong mixing of the virtual benzene and $\mathrm{Cu} 4 p$ orbitals. However, much stronger benzene $\sigma-\pi$ mixing is perceived at the CASSCF level, owing, most 
likely to the neglect of dynamical electron correlation in CASSCF. It appears that some of the possible metal to benzene-triplet bonding interactions are directly included as some of the excitations available within the active space. All possible interactions may have been included, if the ground-state energy could have been reduced in this process, however, and all interactions are indeed included at the CASPT2 level. Because of the high level of $\sigma-\pi$ mixing, quantification of the significasnce of the benzene triplet states at either the CASSCF or CASPT2 levels is difficult, although it is clear that they do not dominate the binding.

The interaction energy of benzene and the cluster calculated by VASP DFT is -19 $\mathrm{kcal} \mathrm{mol}^{-1}$, some $14 \mathrm{kcal} \mathrm{mol}^{-1}$ less than the CASPT2 value; the correlation energy from the DFT calculation is thus perceived to be ca. $-57 \mathrm{kcal} \mathrm{mol}^{-1}$ or only $80 \%$ of that determined by CASPT2. While core-valence correlation energies are well represented by DFT, ${ }^{6}$ a major cause of the underestimation of the binding energies is the inadequate treatment of dispersion forces offered by all currently available density functions. ${ }^{57}$ These forces dominate weakly interacting systems such as physisorbed adsorbates on solid surfaces, and, whether by design or otherwise, are accounted for as part of a perceived covalent bonding interaction. ${ }^{38}$ Consequently, the quality of DFT predictions for weakly bound systems varies dramatically, possibly either underestimating or overestimating binding energies by an order of magnitude. Dispersion contributions to strong interactions involving covalent binding are usually of the same magnitude as those to physisorbed interactions, but as the covalent forces are much larger, improper treatment of dispersion does not present a critical problem. While there have been attempts to incorporate realistic descriptions of dispersive interactions empirically within DFT, ${ }^{66 ; 69 ; 70}$ such refinements are not yet applicable or well characterized for practical purposes.

While the CASPT2 calculations clearly indicate the major qualitative features controlling the binding, accurate quantitative calculations at this level are dif- 
ficult to perform. Basis sets of the size used herein are generally considered to give results of accuracy of ca. $5 \mathrm{kcal} \mathrm{mol}^{-1}$ for second-row complexes; however, the number of electrons retained in the calculation, and the extent of electron correlation within the metal bands, can have profound effects on the accuracy of the calculation.

A simple test that verifies that the active space used is not unrealistic is provided by an MP2 calculation using only a single spin-adapted reference determinant. The calculated MP2 interaction energy is $-26 \mathrm{kcal} \mathrm{mol}^{-1}$, quite close to the CASPT2 value of $-33 \mathrm{kcal} \mathrm{mol}^{-1}$. Previous MP2 calculations on this system ${ }^{20}$ have predicted either no binding or weak binding, is contrast to this result. In the current calculations a 10-electron effective core potential is used, explicitly including 19 electrons per copper atom in the calculations, compared to 1 - 11 electrons included previously. The dispersion and core-valence correlation energies are proportional to the number of nearby electrons, with the effect of reducing the number of electrons per atom to 11 being enhanced by the inclusion of only 6 copper atoms in the earlier calculations. It is thus anticipated that the inclusion of 13 atoms containing 19 electrons in the present calculations could approximate the asymptotic limit. However, the core-valence correlation energy is accounted for in the present calculations at the CASPT2 level, and as these contributions to the binding may be significant, ${ }^{68}$ enhanced quantitative accuracy would be expected if the $3 d$ orbitals were included in the active space.

A simple test for the adequacy of the basis set is the magnitude of the BSSE correction. At $26 \mathrm{kcal} \mathrm{mol}^{-1}$, this correction is quite large. Even the adequacy of the application of the BSSE correction on systems of this type using sufficiently large basis sets, of the sized used herein, has been questioned ${ }^{49}$ as it may change calculated binding energies in the wrong direction or double the actual effect. ${ }^{50}$ Clearly, much larger basis sets ${ }^{49}$ are required in quantitative calculations. 


\section{Conclusions}

The typically strong interaction of benzene with surfaces of transition metals has previously been extensively studied owing to its technological relevance. Here, the nature of the adsorption of benzene to the coinage metals copper, silver and gold is shown to be significantly different with the adsorbate only weakly interacting with the surfaces. For benzene on $\mathrm{Cu}(111), \operatorname{Ag}(111)$, and $\mathrm{Au}(111)$ the binding is clearly identified as being weak and physisorptive, with all major qualitative features of the available experimental results being reproduced by the calculations. A major quantitative feature not properly predicted is the magnitude of the binding energy, a quantity that is dramatically underestimated, however. For benzene on $\mathrm{Cu}(110)$, a variety of feasible physisorption-like and chemisorption-like structures are predicted. Owing to the underestimation of the binding energies, authoritative discrimination between these possibilities based solely on calculated energies is not feasible. However, the calculated PDOS of the various structures are shown to differ significantly, and the chemisorbed-like ones actually appear to give the best agreement with experimental results. Nevertheless, neither the possibility that the physisorbed structures prevail, nor the possibility that zero-point and thermal fluctuations dominate by mixing the structures, can be eliminated. Previously, the prominence of highly distorted chemisorbed structures for benzene on $\mathrm{Cu}(110)$ had been anticipated through model DFT calculations of benzene on a $\mathrm{Cu}_{13}$ cluster, ${ }^{21 ; 22}$ but our analogous calculations for that system and for benzene on a periodic surface indicate that the model cluster is too reactive for use in quantitative studies of adsorption structure and energetics. The predicted PDOS for these cluster-optimized distorted structures are inconsistent with the available experimental information and are hence excluded from contention for the structure of benzene on $\mathrm{Cu}(110)$.

A significant feature of the calculated binding between benzene and the (110) 
and (111) surfaces is that covalent bonding contributions are insignificant. Instead, the interactions are dominated by dispersive forces. Calculations of the interaction of benzene with the $\mathrm{Cu}_{13}$ cluster used to model the $\mathrm{Cu}(110)$ surface provide quantitative support to this conclusion: no binding at all is predicted between benzene and the cluster at the CASSCF level, whilst CASPT2 calculations reveal a massive intermolecular correlation energy. In addition, spin-uncoupling models ${ }^{21}$ that anticipate strong covalent interactions between $\mathrm{Cu}(110)$ and the lowest triplet excited state of benzene are shown to be inappropriate by both the DFT calculations, which indicate that the ground-state of the adsorbate correlates to the ground-states of the separated species, and by the CASPT2 calculations, calculations that explicitly include all possible states of bond preparedness of the cluster. The DFT calculations hint that spin-uncoupling may become quite significant for other systems with stronger metal-alkene interactions, however. It is the preeminence of dispersive forces in the benzene - $\mathrm{Cu}(110)$ interacton that leads to the previously noted very poor quantitative predictions of binding strengths by DFT methods.

The authoritative prediction of the structure and properties of aromatic molecules interacting through $\pi$-stacking interactions with surfaces of copper, silver, and gold is thus shown to be a very difficult task that is not currently feasible. Such calculations must include proper treatment of the periodic metallic surfaces, accurate treatment of the dispersive forces between the surface and molecule, and adequate treatment of quantum molecular motion. DFT-based methods recognize that dispersive forces modulate the DOS of the system but fail to include the contribution of that modulation to the total energy. Hence these methods may be of greater use in determining electronic properties than in predicting structural equilibria, but such use of DFT remains limited by intrenched problems such as band-lineup error and band-gap error. 


\section{Acknowledgments}

The work was supported by the Australian Research Council. The use of computer facilities at the Australian Partnership for Advanced Computing (APAC) and Australian Centre for Advanced Computing and Communications (AC3) is gratefully acknowledged. 


\section{References}

[1] Somorjai, G.A. Introduction to Surface Chemistry and Catalysis; Wiley: New York, 1994.

[2] Tour, J.M.; Jones II, L.; Pearson, D.L.; Lamba, J.J.S.; Burgin, T.P.; Whitesides, G.M.; Allara, D.L.; Parikh, A.N.; Atre, S.V. J. Am. Chem. Soc. 1995, 117, 9529-34.

[3] Reed, M.A.; Zhou, C.; Muller, C.J.; Burgin, T.P.; Tour, J.M. Science 1997, 278, 252-4.

[4] Ulman, A. Chem. Rev. 1996, 96, 1533-54.

[5] Lavrich, D.J.; Wetterer, S.M.; Bernasek, S.L.; Scoles, G. J. Phys. Chem. $B$ 1998, 102, 3456-65.

[6] Grönbeck, H.; Curioni, A.; Andreoni, W. J. Am. Chem. Soc. 2000, 122, 3839-42.

[7] Andreoni, W.; Curioni, A.; Grönbeck, H. Int. J. Quantum Chem. 2000, $80,598-42$.

[8] Gottschalck, J.; Hammer, B. J. Chem. Phys. 2002, 116, 784-90.

[9] Bilić, A.; Reimers, J.R.; Hush, N.S. J. Chem. Phys. 2005, 122, 094708$1-15$.

[10] Crossley, M.J.; Prashar, J.K. Tetrahedron Lett. 1997, 38, 6751-4.

[11] Reimers, J.R.; Hall, L.E.; Crossley, M.J.; Hush, N.S. J. Phys. Chem. A 1999, 103, 4385-97.

[12] Bilić, A.; Reimers, J.R.; Hush, N.S. J. Phys. Chem. B 2002, 106, 6740-7.

[13] Chesters, M.A.; Somorjai, G.A. Surf. Sci. 1975, 52, 21-8.

[14] Wöll, C. J. Synch. Rad. 2001, 8, 129-35.

[15] Dudde, R.; Frank, K-H.; Koch, E-E. Surf. Sci. 1990, 225, 267-72.

[16] Netzer, F.P. Langmuir 1991, 7, 2544-7.

[17] Xi, M.; Yang, M.X.; Jo, S.K.; Bent, B.E.; Stevens, P. J. Chem. Phys. 1994, 101, 9122-31.

[18] Lukes, S.; Vollmex, S.; Witte, G.; Wöll, C. J. Chem. Phys. 2001, 114, 
10123-30.

[19] Lomas, J.R.; Baddeley, C.J.; Tikhov, M.S.; Lambert, R.M. Langmuir 1995, 11, 3048-53.

[20] Lomas, J.R.; Pacchioni, G. Surf. Sci. 1996, 365, 297-309.

[21] Triguero, L.; Pettersson, L.G.M.; Minaev, B.; Ågren, H. J. Chem. Phys. 1998, 108, 1194-205.

[22] Pettersson, L.G.M.; Ågren, H.; Luo, Y.; Triguero, L. Surf. Sci. 1998, 408, $1-20$.

[23] Rogers, B.L.; Shapter, J.G.; Ford, M.J. Surf. Sci. 2004, 548, 29-40.

[24] Møller, C.; Plesset, M.S. Phys. Rev. A 1934, 46, 618-22.

[25] Andersson, K.; Malmqvist, P.-A.; Roos, B.O. J. Chem. Phys. 1992, 96, 1218-26.

[26] Kresse, G.; Hafner, J. Phys. Rev. B 1993, 47, 558-61.

[27] Kresse, G.; Furthmüller, J. Comput. Mat. Sci. 1996, 6, 15-30.

[28] Segall, M.D.; Lindan, P.J.D.; Probert, M.J.; Pickard, C.J.; Hasnip, P.J.;

Clark, S.J.; Payne, M.C. J. Phys.: Condens. Matt. 2002, 14, 2717-44.

[29] Ordejón, P.; Artacho, E.; Soler, J. M. Phys. Rev. B 1996, 53, R10441-44.

[30] Soler, J.M.; Artacho, E.; Gale, J.D.; García, A.; Junquera, J.; Ordejón, P.; Sánchez-Portal, D. J. Phys.: Condens. Matt. 2002, 14, 2745-79.

[31] Vanderbilt, D. Phys. Rev. B 1990, 41, 7892-5.

[32] Kresse, G.; Hafner, J. J. Phys. Condens. Matter 1994, 6, 8245-57.

[33] Perdew, J.P.; Wang, Y. Phys. Rev. B 1992, 45, 13244-9.

[34] Perdew, J.P.; Burke, W.; Ernzerhof, M. Phys. Rev. Lett. 1996, 77, 3965-8.

[35] Troullier, N.; Martins, J.L. Phys. Rev. B 1991, 43, 1993-2006.

[36] Boys, S.F.; Benardi, F. Mol. Phys. 1970, 19, 553-7.

[37] Neugebauer, J.; Scheffler, M. Phys. Rev. B 1992, 46, 16067-80.

[38] Bilić, A.; Reimers, J.R.; Hush, N.S.; Hafner, J. J. Chem. Phys. 2002, $116,8981-7$.

[39] Methfessel, A.; Paxton, A.T. Phys. Rev. B 1989, 40, 3616-21.

[40] Burns, W.J.; van der Waals, J.H.; van Hernerd, M.C. J. Am. Chem. Soc. 
1989, 111, 86-7.

[41] Andersson, K.; Blomberg, M.R.A.; Fülscher, M.P.; Karlström, G.; Lindh, R.; Malmqvist, P.-Å.; Neogrády, P.; Olsen, J.; Roos, B.O.; Sadlej, A.J.; Seijo, L.; Serrano-Andrés, L.; Siegbahn, P.E.M.; Widmark, P.-O. Molcas Version 4; University of Lund: Lund, 1997.

[42] Andrae, D.; Häußermann, U.; Dolg, M.; Stoll, H.; Preu $\beta$, H. Theor. Chim. Acta 1990, 77, 123-41.

[43] Hehre, W.J.; Ditchfield, R.; Pople, J.A. J. Chem. Phys. 1972, 56, 225761.

[44] Frisch, M.J.; Trucks, G.W.; Schlegel, H.B.; B2, et al. GAUSSIAN 03 Rev.; Gaussian Inc.: Pittsburgh PA, 2003.

[45] Chatterjee, R.; Postawa, Z.; Winograd, N.; Garrison, B.J. J. Phys. Chem. $B$ 1999, 103, 151-63.

[46] Anderson, A.B.; McDevitt, M.R.; Urbach, F.L. Surf. Sci. 1984, 146, 8092.

[47] Doering, M.; Rust, H.-P.; Briner, B.G.; Bradshaw, A.M. Surf. Sci. 1988, 410, L736-40.

[48] Komeda, T.; Kim, Y.; Fujita, Y.; Sainoo, Y.; Kawai, M. J. Chem. Phys. 2004, 120, 5347-52.

[49] Lambropoulos, N.A.; Reimers, J.R.; Hush, N.S. J. Chem. Phys. 2002, 116, 10277-86.

[50] Cai, Z.-L.; Reimers, J.R. J. Phys. Chem. A 2002, 106, 8769-78.

[51] Zhou, X.-L.; Castro, M.E.; White, J.M. Surf. Sci. 1990, 238, 215-25.

[52] Munakata, T.; Shudo, K. Surf. Sci. 1999, 433, 184-7.

[53] Petterson, L.G.M.; Bagus, P.S. Phys. Rev. Lett. 1986, 56, 500-3.

[54] Michaelides, A.; Hu, P.; Lee, M.-H.; Alavi, A.; King, D.A. Phys. Rev. Lett. 2003, 90, 246103-1-4.

[55] Migani, A.; Sousa, C.; Illas, F. Surf. Sci. 2005, 574, 297-305.

[56] Becker, R.S.; Wentworth, W.E. J. Am. Chem. Soc. 1963, 85-4.

[57] Reimers, J.R.; Cai, Z.-L.; Bilić , A.; Hush, N.S. Ann. N.Y. Acad. Sci. 
2003, 1006, 235-51.

[58] Weinelt, M.; Wassdahl, N.; Weill, T.; Karis, O.; Hasselström, J.; Bennich, P.; Nilsson, A. Phys. Rev. B 1998, 7351-60.

[59] Triguero, L.; Föhlisch, A.; Väterlein, P.; Hasselström, J.; Weinelt, M.; Pattersson, L.G.M.; Luo, Y.; Ågren, H.; Nilsson, A. J. Am. Chem. Soc. 2000, 122, 12310-6.

[60] Triguero, L.; Luo, Y.; Pettersson, L.G.M.; Ågren, H. Phys. Rev. B 1999, 5189-200.

[61] Mittendorfer, F.; Hafner, J. Surf. Sci. 2001, 472, 133-5.

[62] Frank, K. H.; Dudde, R.; Koch, E.E. Chem. Phys. Lett. 1986, 132, 83-7.

[63] Reimers, J.R.; Hall, L.E.; Hush, N.S.; Silverbrook, K. Ann. N.Y. Acad. Sci. 1998, 852, 38-53.

[64] Reimers, J.R.; Hush, N.S. Inorg. Chim. Acta 1994, 226, 33-42.

[65] Reimers, J.R.; Shapley, W.A.; Hush, N.S. J. Chem. Phys. 2003, 119, $3240-8$.

[66] Zhechkov, L.; Heine, T.; Patchkovskii, S.; Seifert, G.; Duarte, H.A. J. Chem. Theory and Comput. 2005, 1, 841-7.

[67] Müller, M.; Meyer, W. J. Chem. Phys. 1984, 80, 3311-20.

[68] Triguero, L.; Wahlgren, U.; Pettersson, L.G.M.; Siegbahn , P..

[69] Wu, X.; Vargas, M.C.; Nayak, S.; Lotrich, V.; Scoles, G. J. Chem. Phys. 2001, 115, 8748-8757.

[70] Xu, X.; Goddard III, W.A. Proc. Natl. Acad. Sci. U.S.A. 2004, 101, 2673-7. 


\section{Table 1}

Adsorption energy changes $\Delta E$ as calculated by VASP for the $\mathrm{C}_{6} \mathrm{H}_{6^{-}}(3 \times 3)-\mathrm{M}(111)$ system, where $\mathrm{M}=\mathrm{Cu}, \mathrm{Ag}$, and $\mathrm{Au}$, given in $\mathrm{kcal} \mathrm{mol}^{-1}$, for respective molecule conformations. Positive values indicate endothermic reactions and are obtained as the calculations terminate simply when the forces generated are smaller than a preselected limit and the potential-energy surfaces are very flat.

\begin{tabular}{ccccccccc}
\hline \hline $\mathrm{M}$ & TOP-A & TOP-B & BR-A & BR-B & FCC-A & FCC-B & HCP-A & HCP-B \\
\hline $\mathrm{Cu}$ & 0.22 & 1.02 & -0.48 & -0.36 & -0.39 & -0.54 & -0.42 & -0.60 \\
$\mathrm{Ag}$ & 0.00 & -0.02 & -1.02 & -0.99 & -1.23 & -1.09 & -1.13 & -1.13 \\
$\mathrm{Au}$ & -0.50 & -0.60 & -1.75 & -1.32 & -1.86 & -1.51 & -1.92 & -1.63 \\
\hline \hline
\end{tabular}


Table 2

Calculated adsorption energy changes $\Delta E$ for $\mathrm{C}_{6} \mathrm{H}_{6^{-}}(2 \times 3)$ on $\mathrm{Cu}(110)$, given in kcal $\mathrm{mol}^{-1}$, for the adsorbate orientations illustrated in Fig. 2, obtained using VASP and SIESTA (with and without correction for BSSE) with the PW91 and PBE density functionals, at starting intermolecular-only optimized geometries and full optimized ones.

\begin{tabular}{|c|c|c|c|c|c|c|c|c|c|}
\hline method & asorbate & TOP-A & TOP-B & SB-A & SB-B & HOL-A & HOL-B & LB-A & LB-B \\
\hline \multirow[t]{3}{*}{ VASP-PW91 } & flat & -1.55 & -1.75 & -4.33 & -4.81 & -4.93 & -5.81 & -5.28 & -4.93 \\
\hline & quinonoid & $a$ & $a$ & $a$ & $a$ & -2.87 & $b$ & -4.35 & $b$ \\
\hline & H-flipped & $a$ & $a$ & $a$ & $a$ & $c$ & -5.19 & $c$ & -3.33 \\
\hline SIESTA-PBE & flat & -1.0 & -1.2 & -1.6 & -1.5 & -2.1 & -2.1 & -1.8 & -1.7 \\
\hline \multirow[t]{2}{*}{ Start, BSSE } & quinonoid & 1.6 & 1.4 & 1.1 & 0.5 & -0.1 & 0.1 & -0.4 & -1.2 \\
\hline & H-flipped & 10.6 & 10.4 & 8.7 & 10.3 & 1.2 & -0.4 & 4.7 & 4.1 \\
\hline SIESTA-PBE & flat & -7.0 & -7.1 & -13.8 & $c$ & -8.9 & $b$ & $c$ & $b$ \\
\hline \multirow[t]{2}{*}{ Opt, Raw } & quinonoid & $a$ & $a$ & $a$ & -13.7 & $b$ & $b$ & -14.2 & $b$ \\
\hline & H-flipped & $a$ & $a$ & $a$ & $c$ & -16.3 & -18.5 & $c$ & -12.8 \\
\hline SIESTA-PBE & flat & -1.0 & -1.2 & 0.6 & $c$ & -1.9 & $b$ & $c$ & $b$ \\
\hline \multirow[t]{2}{*}{ Opt, BSSE } & quinonoid & $a$ & $a$ & $a$ & 0.0 & $b$ & $b$ & 0.4 & $b$ \\
\hline & H-flipped & $a$ & $a$ & $a$ & $c$ & 0.8 & -2.7 & $c$ & 1.7 \\
\hline
\end{tabular}

${ }^{a}$ Collapses to the flat structure upon optimization.

${ }^{b}$ Collapses to the H-flipped structure upon optimization.

${ }^{c}$ Collapses to the quinonoid structure upon optimization. 
Table 3

Calculated properties for VASP PW91-calculated $\mathrm{C}_{6} \mathrm{H}_{6^{-}}(2 \times 3)$ on $\mathrm{Cu}(110): \Delta z$ is the average height of $\mathrm{C}$ above $\mathrm{Cu}, \Delta R_{C C}$ is the maximum difference in $\mathrm{CC}$ bond lengths, $\tau_{C C C C}$ and $\tau_{C C C H}$ are maximum torsion angles, $\Delta \epsilon_{H}-E_{F}$ is the shift in the HOMO orbital energy from the Fermi energy, $\Delta \epsilon_{L}^{g a s}$ is the shift in LUMO energy from the gas phase, $\Delta \epsilon_{L}^{111}$ is the shift in LUMO energy from that for adsorption on $\mathrm{Cu}(111)$, and $q_{m o l}$ is the adsorbate charge from SIESTA Mulliken orbital analysis.

\begin{tabular}{|c|c|c|c|c|c|c|c|c|c|}
\hline adsorbate & structure & $\Delta z$ & $\Delta R_{C C}$ & $\tau_{C C C C}$ & $\tau_{C C C H}$ & $\epsilon_{H}-E_{F}$ & $\Delta \epsilon_{L}^{g a s}$ & $\Delta \epsilon_{L}^{111}$ & $q_{\text {mol }}$ \\
\hline \multirow[t]{8}{*}{ flat } & TOP-A & 2.86 & .002 & .2 & -2.1 & -3.11 & -.96 & -.57 & -.01 \\
\hline & TOP-B & 2.81 & .004 & .4 & -2.4 & -3.11 & -.96 & -.57 & -.01 \\
\hline & SB-A & 2.74 & .005 & .7 & .7 & -3.41 & -1.00 & -.61 & .01 \\
\hline & SB-B & 2.74 & .006 & .7 & 1.2 & -3.41 & -1.00 & -.61 & .00 \\
\hline & HOL-A & 2.71 & .004 & -.5 & 1.6 & -3.48 & -1.17 & -.77 & -.02 \\
\hline & HOL-B & 2.70 & .006 & -.9 & -2.4 & -3.52 & $-1.57,-1.17$ & $-1.18,-.78$ & .01 \\
\hline & LB-A & 2.72 & .008 & .9 & 1.6 & -3.49 & -1.21 & -.82 & .00 \\
\hline & LB-B & 2.73 & .007 & -1.0 & -2.3 & -3.46 & -1.10 & -.70 & -.01 \\
\hline \multirow[t]{3}{*}{ quinonoid } & $\mathrm{HOL}_{-\mathrm{A}^{a}}$ & 2.00 & .062 & 7.4 & 16.0 & -7.27 & -2.06 & -1.67 & .09 \\
\hline & HOL-A & 2.20 & .002 & 1.0 & 8.5 & -7.27 & -1.69 & -1.30 & .05 \\
\hline & LB-A & 2.41 & .011 & 2.0 & 4.0 & -3.98 & -1.52 & -1.13 & .02 \\
\hline \multirow[t]{3}{*}{ H-flipped } & HOL-B ${ }^{a}$ & 2.09 & .011 & -4.3 & 15.9 & -7.21 & -2.62 & -2.23 & .12 \\
\hline & HOL-B & 2.25 & .019 & -1.8 & 9.0 & -4.29 & -1.50 & -1.11 & .09 \\
\hline & LB-B & 2.38 & .020 & 1.9 & 6.4 & -4.02 & -1.37 & -.98 & -.01 \\
\hline
\end{tabular}

${ }^{a}$ embodies the optimized geometry of benzene on a $\mathrm{Cu}_{13}$ cluster ${ }^{22}$ 


\section{Figure Captions}

Fig. 1. Starting geometries for benzene adsorbed on the (111) faces of $\mathrm{Cu}, \mathrm{Ag}, \mathrm{Au}$.

Fig. 2. Initial geometries for benzene adsorbed on the $\mathrm{Cu}(110)$ surfaces.

Fig. 3. The structures of benzene considered for the adsorption on $\mathrm{Cu}(110)$ : (a) planar, (b) quinonoid, ${ }^{22}$ and (c) H-flipped. ${ }^{22}$

Fig. 4. VASP PW91 calculated average carbon $p_{z}(\pi)$ density of states $\rho$ as a function of the orbital energy difference from the Fermi energy, $E-E_{F}$, for gas-phase benzene, benzene on $\mathrm{Cu}(111)$, and benzene on $\mathrm{Cu}(110)$ in the flat, quinonoid, and H-flipped structures; (solid)- fully optimized structures on the surface, (dashed)starting structures based on cluster-optimized geometries. ${ }^{22}$

Fig. 5. VASP PW91 calculated average copper $d_{z^{2}}$ density of states $\rho$ as a function of the orbital energy difference from the Fermi energy, $E-E_{F}$, for a clean surface, benzene on $\mathrm{Cu}(111)$, and benzene on $\mathrm{Cu}(110)$ in the flat, quinonoid, and H-flipped structures; (solid)- fully optimized structures on the surface, (dashed)starting structures based on cluster-optimized geometries. ${ }^{22}$

Fig. 6. Deconvolution of the observed ${ }^{62}$ inverse photoemission spectra of a clean $\mathrm{Cu}(111)$ surface and surface with benzene adsorbed into Gaussian-shaped bands on a piecewise-linear background.

Fig. 7. Fitting of the observed ${ }^{48}$ STM current as a function of voltage (the energy above the Fermi energy) for benzene on $\mathrm{Cu}(110)$, revealing molecular resonances centred at 3.0, 3.7, and $4.5 \mathrm{eV}$. 
Fig. 8. The DFT calculated ground-state potential-energy surface ) for benzene approaching the $\mathrm{Cu}_{13}$ cluster at minimum carbon to copper surface-plane separation $\Delta z$, along with the calculated surface for the lowest triplet state $(\bullet)$ of benzene, compared to the generic form ${ }^{21}$ of these two surfaces ( - - ) expected in the scenario that a covalent bond is formed between the two species. 


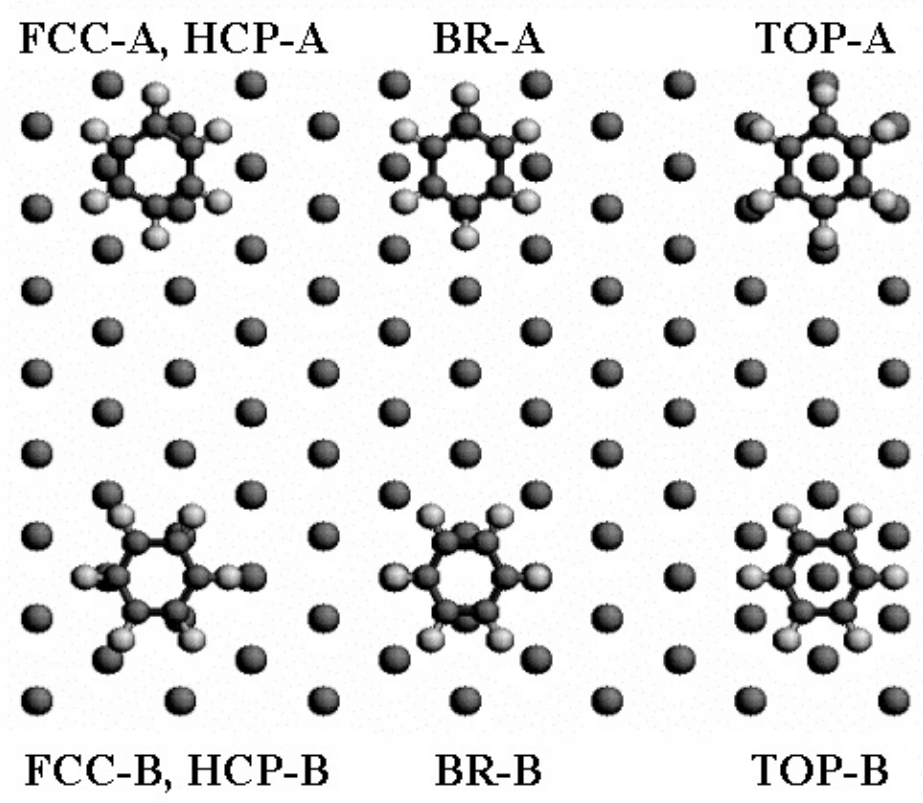

Figure 1 


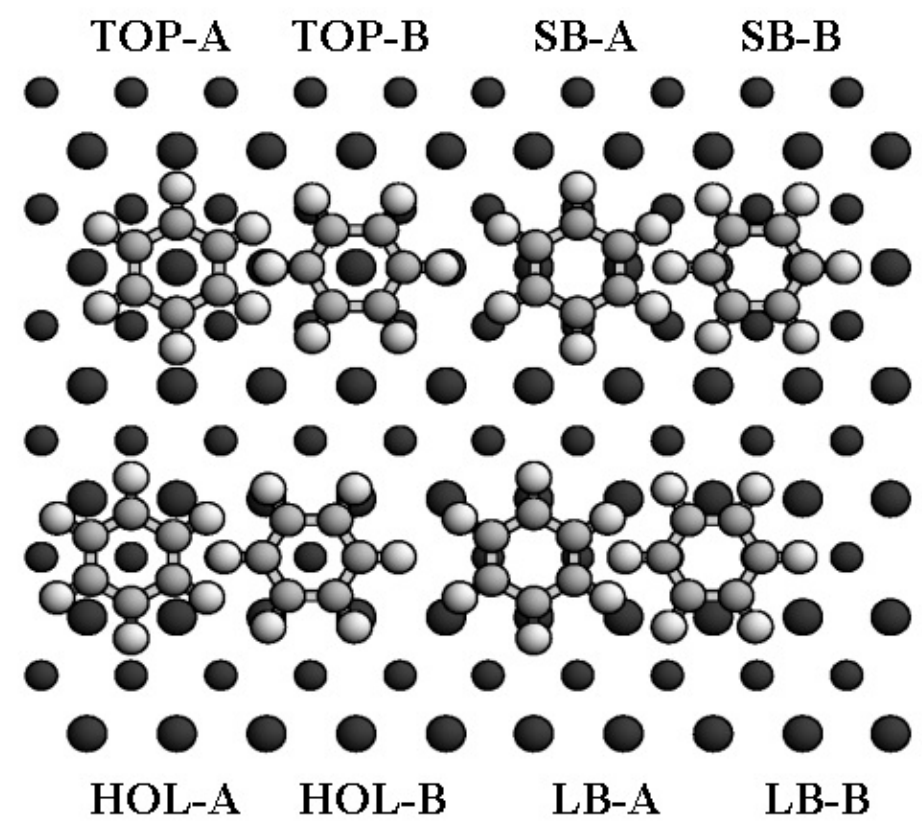

Figure 2 
a) $\mathrm{c}-\mathrm{0}=30$

b) $\quad \mathrm{a}-3=3-3$

c) $\mathrm{a}-\mathrm{a} s=3$

Figure 3 


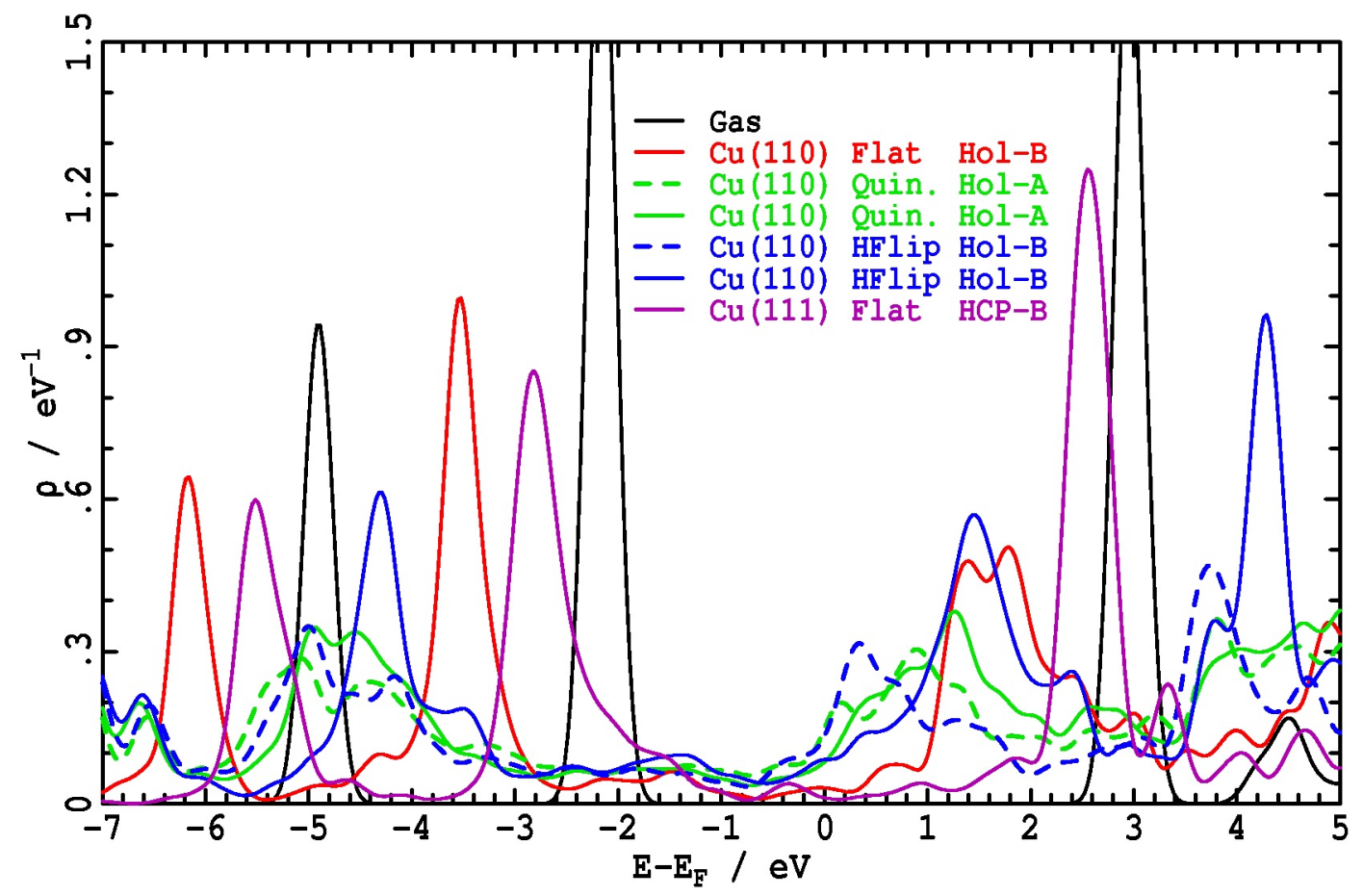

Figure 4: 


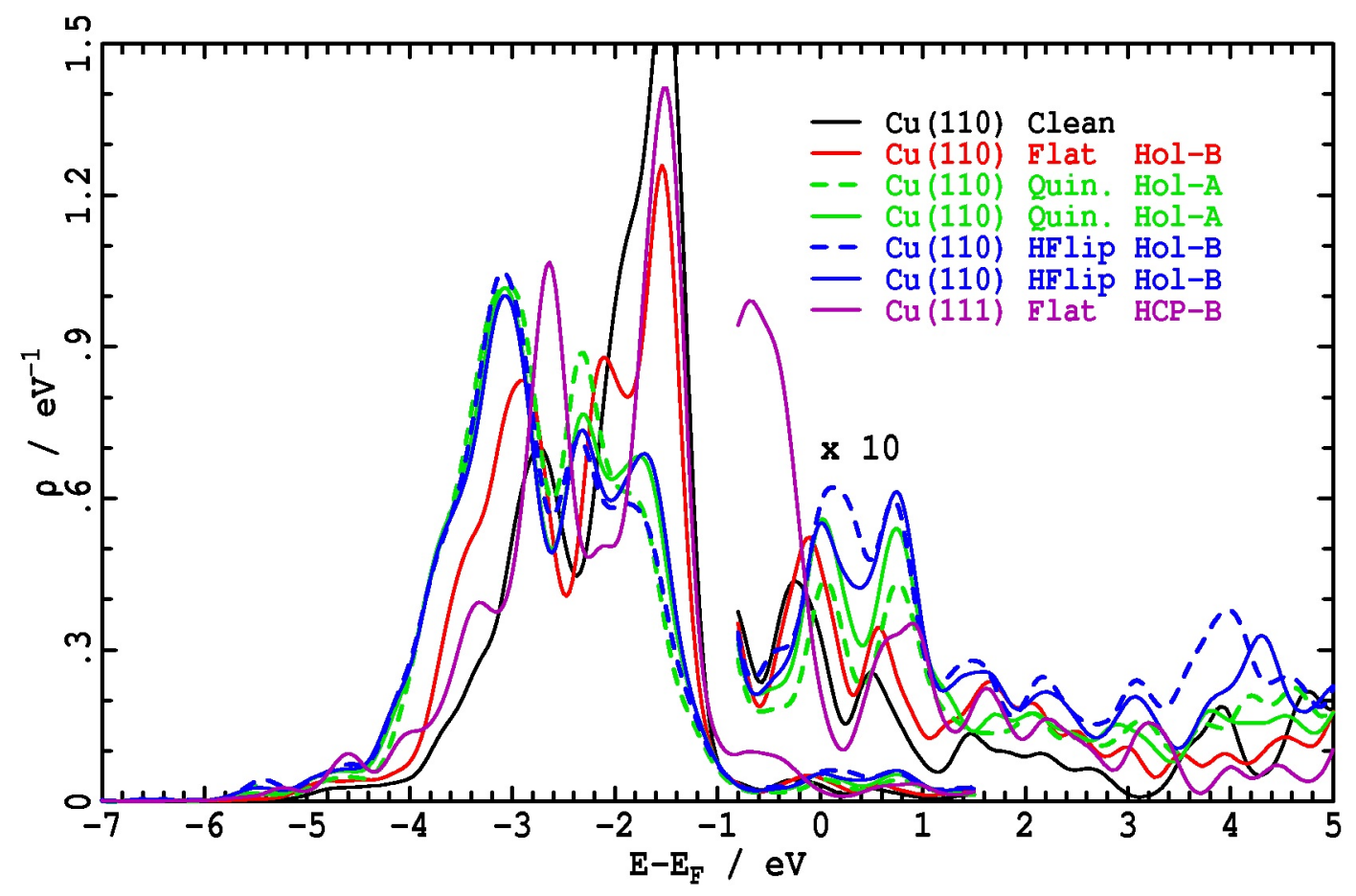

Figure 5: VASP PW91 calculated average bound copper $d_{z 2}$ density of states $\rho$ as a function of the orbital energy difference from the Fermi energy, $\mathrm{E}-\mathrm{E}_{\mathrm{F}}$, for a clean surface, benzene on $\mathrm{Cu}(111)$, and benzene on $\mathrm{Cu}(110)$ in the flat, quinonoid, and H-flipped structures; (solid)- fully optimized structures on the surface, (dashed)- starting structures based on cluster-optimized geometries lcite $\{$ palt98\}. 


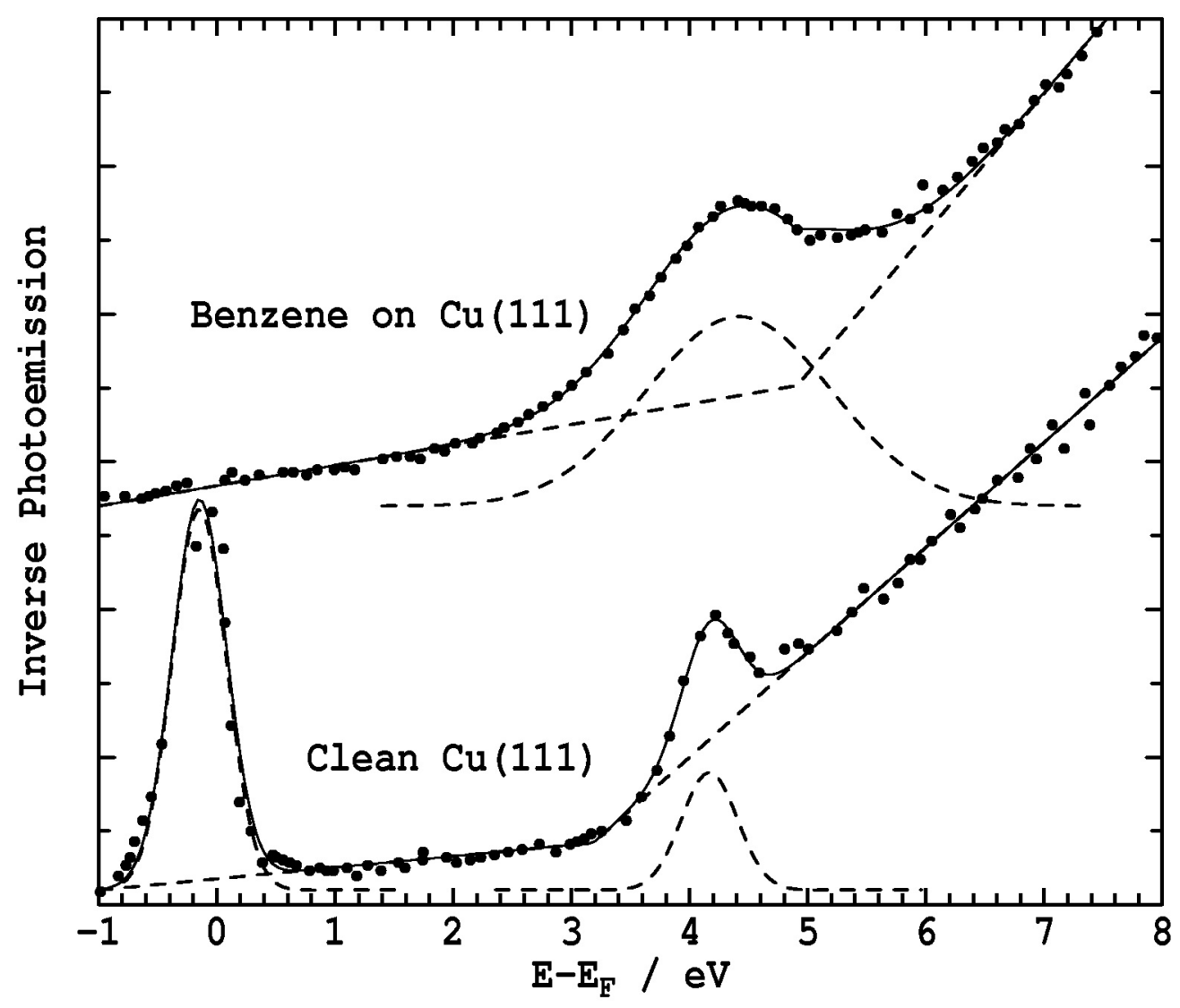

Figure 6: Deconvolution of the observed $\backslash \operatorname{cite}\{\mathrm{fdk} 86\}$ inverse photoemission spectra of a clean $\mathrm{Cu}(111)$ surface and surface with benzene adsorbed into Gaussian-shaped bands on a piecewiselinear background. 


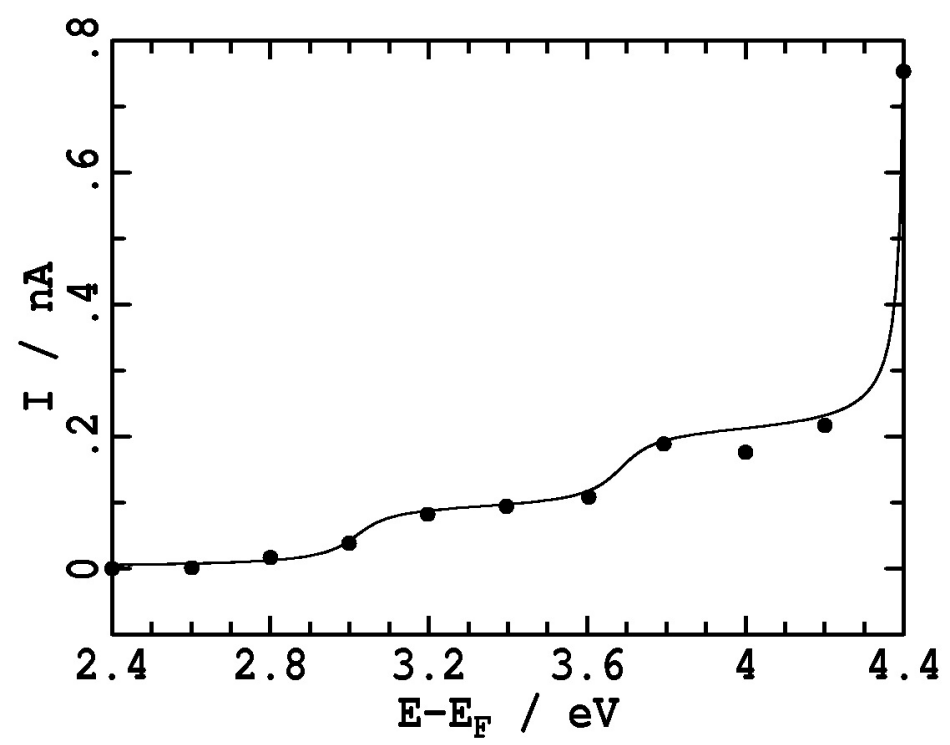

Figure 7: 


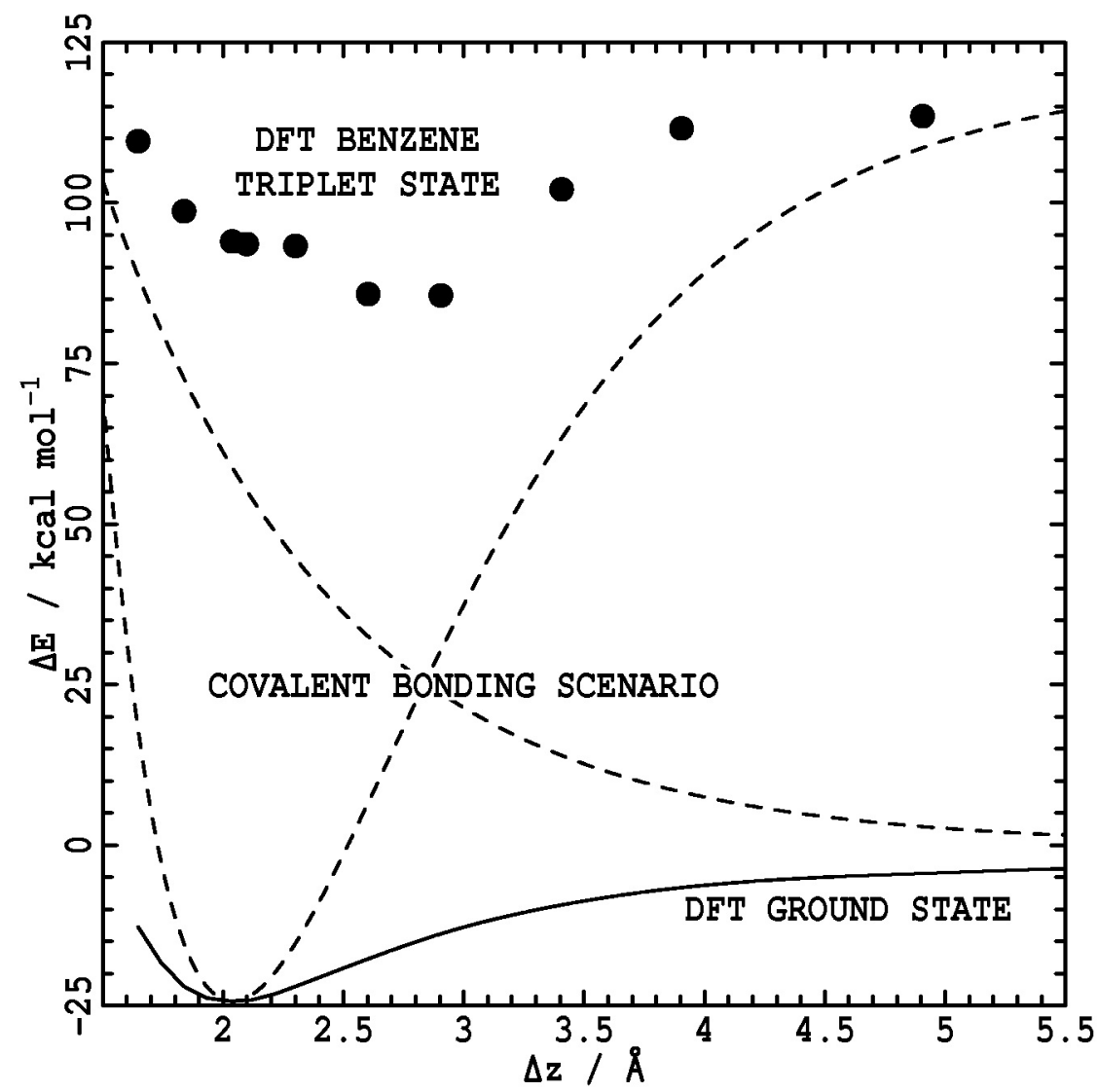

Figure 8: 